\title{
Effect of Stabilization Exercise on Pain and Quality of Life of Patients with Non-specific Chronic Low Back Pain
}

\author{
Akodu, A.K., Tella, B.A., Olujobi, O.D. \\ Department of Physiotherapy, College of Medicine, University of Lagos, PMB 12003, Surulere, Lagos, Nigeria \\ Correspondence \\ A.K. Akodu, Department of Physiotherapy, College of Medicine, University of Lagos, PMB 12003, Surulere, \\ Lagos, Nigeria·E-mail: akoduashiyatkehinde@yahoo.co.uk, aakodu@unilag.edu.ng
}

\begin{abstract}
SUMMARY
Patients with chronic low back pain have lower quality of life compared to patients with other chronic diseases and the general population. This study was designed to assess the effect of stabilization exercises on the quality of life of patients with non-specific chronic low back pain (NSCLBP).

A total of 23 patients with NSCLBP, aged 27-65 years, participated in this study. They were recruited from the Orthopaedic Clinic of Lagos University Teaching Hospital (LUTH), Lagos, Nigeria. They were put through core stabilization exercises twice weekly for 4 consecutive weeks and assessed with the World Health Organization Quality of Life (WHO QoL) questionnaire and Verbal Rating Scale (VRS) at baseline and at four weeks post-treatment intervention. Ethical approval was sought and obtained from the Health Research and Ethics Committee of LUTH. Data was analysed using the Statistical Package for Social Science (SPSS) version 17 , and the level of significance was set at $\mathrm{p}<0.05$.

Patients recorded significant reduction in pain severity following intervention $(p=0.01)$. There was also significant improvement in the quality of life domains (psychological health and social relationship) $(p=0.01)$ post intervention, and only age correlated directly with social relationship of the quality of life domain $(r=0.59$, $\mathrm{p}=0.01)$.

This study concluded that stabilization exercises reduced pain and improved the psychological health as well as social relationship domains of the quality of life of patients with non-specific chronic low back pain.
\end{abstract}

KEY WORDS: stabilization, quality of life, non-specific low back pain

\section{INTRODUCTION}

Low back pain (LBP) is a major health problem which affects up to $80 \%$ of the adult population (Walker et al, 2004) and accounts for considerable healthcare and socioeconomic costs (Gray and Maniadakis, 2000), while nonspecific low back pain (NSLBP) accounts for about $85 \%$ of LBP in patients seen in primary health care settings (Deyo and Philips, 1996).

Patients suffer physical disabilities and psychological distress concurrently with low back pain (Bogduk, 2014). Waddell et al. (1996) described chronic low back pain (CLBP) as a 21 st century epidemic. The prognosis of acute NSLBP is relatively favourable as a significant percentage of sufferers; probably over $50 \%$, do not consult a healthcare professional for the problem because it resolves on its own (Waxman et al., 1998). However, in a small group of acute patients, the problem fails to resolve as it should. Perhaps $10 \%$ go on to develop chronic, disabling LBP (Klenerman et al., 1995; Carey et al., 2000). This disability of patients with CLBP is predicted by pain duration, and the quality of life of such patients is predicted by disability (Kovacs et al., 2005).

Back pain affects the quality of life of its sufferers, particularly with regard to enjoyment of leisure-time activities (Heliovaara et al., 1989), reduction in work production output, difficulty in performing household 
chores (Lamers et al., 2005), sporting activities (Duquesnoy et al., 1998).

Stabilization exercise is aimed at protecting and supporting the spinal segment from re- injury by reestablishing and enhancing muscle control to compensate for any loss of muscle action caused by injury or degenerative changes (Richardson et al., 1999). Gatti et al. (2011) reported that stabilization exercise was found to be effective in reducing disability in patients with CLBP. It was also concluded, in a study by Akodu et al. (2015), that stabilization exercise is effective in the reduction of pain and improvement of functional disability in patients with NSCLBP. In another study by Akodu et al. (2013), it was established that stabilization exercise is effective in increasing the cross-sectional area of the lumbar multifidus muscle, which is one of the muscles needed to maintain proper stability of the spine.

This study was however designed to assess the effect of stabilization exercise on pain and the quality of life of patients with NSCLBP.

\section{MATERIALS AND METHODS}

\section{Participants}

A total of 30 patients (16 males and 14 females) with NSCLBP were recruited for this study from Lagos University Teaching Hospital, Idi-Araba. All the patients included in the study were subjects with a history of NSCLBP with or without pain radiating to one or both lower limbs, and whose clinical assessment indicated that they were suitable for stabilization exercise training. Excluded from the study were: patients confirmed to be pregnant, and patients with specific LBP or with medical or surgical conditions that might hinder exercise performance.

\section{Procedure for Data Collection}

Prior to the commencement of the study, the demographic data of the patients were obtained, such as age, gender, weight, height, occupation, marital status, clinical history of LBP and number of LBP episodes during the 12 months prior to the study. Baseline assessment of pain and quality of life using the verbal rating scale (VRS) and the World Health Organization Quality of Life questionnaire was conducted. The subjects gave their consent by filling an informed consent form. Ethical approval was sought and obtained from the Health Research and Ethics Committee of Lagos University Teaching Hospital, Idi-Araba, Lagos.
Out of the 30 patients, 5 were found ineligible for the study after screening and were therefore excluded. However, only 23 patients (12 males and 11 females) completed the study; two patients did not complete due to illness.

Subjects went through the stabilization exercise protocol twice weekly for 4 consecutive weeks.

\section{Stabilization Exercise Protocol}

Abdominal bracing: Subjects were instructed in supine lying position to perform drawing-in manoeuvre of the abdomen, holding it for 8 seconds, doing 30 repetitions.

Bracing with heel slides: Subjects were instructed in supine lying to perform drawing-in manoeuvre of the abdomen, holding it for 8 seconds, doing 20 repetitions with sliding of each heel for 4 seconds.

Bracing with bridging: Subjects were instructed in supine lying to perform drawing-in manoeuvre of the abdomen, gently raising the buttocks from the supporting surface and holding it for 8 seconds, doing 30 repetitions.

Bracing with leg lift: Subjects were instructed in supine lying position to perform drawing - in manoeuvre of the abdomen, holding it while raising each leg for 4 seconds, doing 20 repetitions.

Bracing with bridging and leg lift: Subjects were instructed in supine lying to perform drawing-in manoeuvre of the abdomen while gently raising the buttocks from the supporting surface, holding this position while raising each leg for 8 seconds, doing 30 repetitions.

Bracing with standing: Subjects were instructed while standing to perform drawing-in manoeuvre of the abdomen for 8 seconds, doing 30 repetitions.

Quadruped arm lift with bracing (one upper limb flexed): Subjects were instructed, while in prone kneeling position to perform drawing-in manoeuvre of the abdomen for 8 seconds, with one upper limb flexed, doing 30 repetitions.

Quadruped leg lift with bracing (one lower limb is extended and lifted off the supporting surface): Subjects were instructed, while in prone kneeling position, to perform the drawing- in manoeuvre of the abdomen with one lower limb flexed for 8 seconds, doing 30 repetitions.

Quadruped alternate arm and leg lift with bracing (contralateral upper and lower limbs are extended alternately): Subjects were instructed, while in prone kneeling position, to perform the drawing-in manoeuvre of 
the abdomen with contralateral upper and lower limbs extended alternately for 8 seconds, doing 30 repetitions (Hicks et al., 2005).

Pain and quality of life assessments were conducted at baseline and at the end of 4 weeks post exercise.

\section{Data Analysis}

The data collected was analysed using SPSS version 17 and was summarized with the descriptive statistics of frequency, mean, standard deviation, and range. Inferential statistics of Chi-square and Wilcoxon's signed rank test were used to compare variables. Results are presented in tables and pie charts. The level of significance of this study was set at $p$ $<0.05$.

\section{RESULTS}

A total of 23 patients with non-specific chronic low back pain (NSCLBP) participated in the study. They comprised $12(52.2 \%)$ males and $11(47.8 \%)$ females, with mean age of $42.30 \pm 12.60$, weight of $76.22 \pm 9.59 \mathrm{~kg}$, height of $1.70 \pm 0.43 \mathrm{~m}$, and a BMI of $26.31 \pm 2.95 \mathrm{~kg} / \mathrm{m}^{2}$.

Baseline and 4 Weeks (Post Exercise) Quality of Life Assessment of the Subjects

The parameters of quality of life (QOL) domains that showed statistically significant differences pre- and posttreatment intervention were psychological stress $(\mathrm{P}=0.01)$ and social relationships $(\mathrm{P}=0.01)$ (table1).

Table 1. Baseline (pre) and 4 weeks (post) quality of life assessment of the subjects

\begin{tabular}{lllll}
\hline QOL & $\begin{array}{l}\text { Pre-treatment } \\
\text { (Baseline) } \\
\text { Mean } \pm \text { SD }\end{array}$ & $\begin{array}{l}\text { Post-treatment } \\
\text { (After 4 weeks) } \\
\text { Mean } \pm \text { SD }\end{array}$ & Z -value & P-value \\
\hline $\begin{array}{l}\text { Domain 1 } \\
\text { (Physical Health) }\end{array}$ & $56.23 \pm 14.63$ & $56.39 \pm 7.27$ & 0.21 & 0.83 \\
$\begin{array}{l}\text { Domain 2 } \\
\text { (Psychological) }\end{array}$ & $64.35 \pm 14.26$ & $72.17 \pm 11.48$ & 3.63 & $0.01^{*}$ \\
$\begin{array}{l}\text { Domain 3 } \\
\text { (Social } \\
\text { relationships) }\end{array}$ & $52.46 \pm 9.70$ & $62.61 \pm 11.32$ & 3.65 & $0.01^{*}$ \\
$\begin{array}{l}\text { Domain 4 } \\
\text { (Environment) }\end{array}$ & $60.87 \pm 11.52$ & $63.04 \pm 10.33$ & 2.74 & 0.06 \\
\hline
\end{tabular}

*Significant at $\mathrm{p}<0.05$

Key: $\mathrm{Z}$ value $=\mathrm{W}$ ilcoxon's signed rank test $; \mathrm{SD}=$ standard deviation; $\mathrm{QOL}=$ Quality of Life
Correlation of Physical Characteristics and Post Treatment (4 Weeks) Assessment of the Subjects' Quality of Life Domains

The physical characteristics of the subjects which include age, height, BMI, and weight, were correlated with posttreatment assessment of quality of life domains. Only age $(\mathrm{p}=0.01)$ showed a statistically significant relationship on correlation with domain 3 (social relationships). The other physical characteristics (weight, height, BMI) did not show any statistically significant relationship on correlation with each parameter of the post-treatment assessment of quality of life domains (psychological, social relationships, and environment) (table 2).

Table 2. Correlation between physical characteristics and quality of life domains

\begin{tabular}{lcllll}
\hline & Correlations & $\begin{array}{l}\text { Domain 1 } \\
\text { post }\end{array}$ & $\begin{array}{l}\text { Domain2 } \\
\text { post }\end{array}$ & $\begin{array}{l}\text { Domain 3 } \\
\text { post }\end{array}$ & $\begin{array}{l}\text { Domain 4 } \\
\text { post }\end{array}$ \\
\hline Age & R & 0.30 & 0.21 & 0.59 & 0.29 \\
& P- value & 0.89 & 0.34 & $0.01^{*}$ & 0.17 \\
Weight & $\mathrm{R}$ & 0.16 & 1.92 & 0.01 & 0.21 \\
& P- value & 0.46 & 0.38 & 0.99 & 0.33 \\
Height & $\mathrm{R}$ & 0.01 & 0.17 & 0.19 & 0.20 \\
& P- value & 0.99 & 0.45 & 0.40 & 0.93 \\
BMI & $\mathrm{R}$ & 0.14 & 0.13 & 0.09 & 0.22 \\
& $\mathrm{P}-$ value & 0.54 & 0.55 & 0.70 & 0.31 \\
\hline
\end{tabular}

* Significant at $\mathrm{p}<0.05$

Key: r: Pearson's correlation coefficient

Domain 1: Physical health; Domain 2: Psychological; Domain 3: Social relationships; Domain 4: Environment

BMI : Body mass index

\section{Baseline and 4 Weeks (Post Exercise) Pain Assessment} of the Subjects

Pain intensity ranged from 4 to 10 at baseline with a mean of $5.78 \pm 1.68$, while at the end of 4 weeks, pain intensity ranged from $0-9$ with a mean of $2.26 \pm 2.28$. The result showed a significant difference $(\mathrm{p}=0.01)$ between the VRS at baseline and after 4 weeks post- intervention assessment (table 3).

Table 3. Baseline and 4 weeks (post exercise) pain assessment of the subjects

\begin{tabular}{|c|c|c|c|c|}
\hline & & Mean \pm SD & $\mathrm{T}$ & P-value \\
\hline \multirow[t]{2}{*}{ Pain Intensity } & Pre-treatment & $5.78 \pm 1.68$ & 11.47 & $0.01 *$ \\
\hline & Post-treatment & $2.26 \pm 2.28$ & & \\
\hline
\end{tabular}

* Significant at $\mathrm{p}<0.05$ 
Correlation Between Pain and Post Intervention Assessment of Quality of Life Domains of the Subjects There was a statistically significant difference between pain and domain three (social relationships), post treatment (4 weeks) assessment of quality of life $(p<0.05)$.

Table 4. Correlation between pain and quality of life domains

\begin{tabular}{llrrrr}
\hline Correlation & $\begin{array}{l}\text { Domain } 1 \\
\text { post }\end{array}$ & $\begin{array}{l}\text { Domain } 2 \\
\text { post }\end{array}$ & $\begin{array}{l}\text { Domain } 3 \\
\text { post }\end{array}$ & $\begin{array}{l}\text { Domain 4 } \\
\text { post }\end{array}$ \\
\hline PAIN & $\mathrm{R}$ & 0.22 & 0.40 & 0.50 & 0.07 \\
& $\mathrm{p}$ - value & 0.30 & 0.06 & $0.02 *$ & 0.73 \\
\hline
\end{tabular}

* Significant at $\mathrm{p}<0.05$

Key:

$\mathrm{r}$ : Pearson's correlation coefficient

Domain 1: Physical health; Domain 2: Psychological; Domain 3: Social relationships; Domain 4: Environment

\section{DISCUSSION}

There was a significant reduction in pain severity between the pre and post-intervention period for the subjects in this study. This supports the result of the study by Akodu et al. (2015), who reported that stabilization exercise alone is effective for reducing pain in non-specific chronic low-back pain patients.

According to the result of this study, there was a statistically significant difference in the psychological aspects of the quality of life of the subjects as well as social status. This improvement could be as a result of the pain reduction ability of the stabilization exercises following proper adherence to the stabilization exercise protocols for four weeks, and the general relaxation effect that follows exercises. This result agrees with the study of Moussouli et al. (2014) who studied the effects of stabilization exercises on health-related quality of life in women with chronic lowback pain for four weeks. They concluded that stabilization exercises improved the quality of life of the subjects studied. It is also in support of the result of the study by Horng et al. (2005) and Schiphorst et al. (2008) who reported that psychological factor and functional status seem to determine quality of life in chronic low-back pain patients.

This study showed a statistically significant relationship between the ages of the subjects and post-treatment (4 weeks) QOL domain 3 (social relationships). This could be as a result of re-establishment of the normal control of the deep spinal muscles which must have been lost due to the increasing age of the patients following degenerative changes. This is in agreement with the findings of the study by Ogunlana et al. (2012) that increasing age adversely affects the health-related quality of life of low-back pain patients.

The findings of this study showed a statistically significant difference between pain and social relationships (quality of life domain 3). This could be the result of an increase in the psychological aspect of the patients QOL. This agrees with the findings of a study by Majtas and Liscacova (2007) that there is a link between pain relief from effective use of analgesia and improvement in several QOL domains, most notably improvement in social functioning.

\section{CONCLUSION}

This study established that stabilization exercises reduced pain and improved psychological health as well as the social relationship domains of the quality of life of patients with non-specific chronic low back pain.

\section{Acknowledgement}

The authors appreciate and acknowledge the patients that participated in this study.

\section{References}

Akodu A.K., Akinbo S.R.A., Odebiyi D.O. 2015. Effect of stabilization exercise on pain and disability in patients with non-specific chronic low back pain. Indian Journal of Physiotherapy and Occupational Therapy 9(2): 175-180.

Akodu A.K., Akinbo S.R.A., Odebiyi D.O. 2013. Effect of stabilization exercise on lumbar multifundus muscle thickness in patients with non-specific chronic low back pain. Indian Journal of Physical Therapy 1(2): 43-46.

Bogduk N. 2014. Management of chronic low back pain. Medical Journal of Australia 180(2): 79-83.

Carey T.S., Garrett J.M. and Jackman A.M.L. 2000. Beyond the good prognosis: Examination of an inception cohort of patients with chronic low back pain. Spine 25(1): 115-120.

Deyo R.A. and Phillips W. 1996. Low back pain: A primary care challenge. Spine 21: 2826-2832.

Duquesnoy B., Allaert F.A. and Verdonc Q.B. 1998. Psychosocial and occupational impact of chronic low back pain. Revue de Rhumatisme (English Edition) 65: 33-40.

Gatti R., Faccendini S., Tettamanti A., Barbero M., Balestri A. and Calori G. 2011. Efficacy of trunk balance exercises for individuals with chronic low back pain: A randomized clinical trial. Journal of Orthopaedics and Sport Physical Therapy 41(8): 542-552.

Heliovara M., Sievers K., Timpivaara O., Maatela J., Knekt P., and Makela M. 1989. Descriptive epidemiology and low back pain. Annals of Medicine 21: 327-333. 
Hick G.E. Fritz J.M., Delitto A. and Mcgill S.M. 2005. Preliminary development of clinical prediction rule for determining which patients with low back pain will respond to a stabilization exercise programme. Archive Physicals Medicine Rehabilitation 86: 1753- 1762.

Horng Y.S., Hwang Y.H., Wu H.C., Liang H.W., Mhe Y.J. and Twu F.C. 2005. Predicting health-related quality of life in patients with low back pain. Spine 30: 551-555.

Klenerman L., Slade P.D., Stanley I.M., Pennie B., Reilly J.P., Atchison L.E., Troup J.D.G. and Rose M.J. 1995. The prediction of chronicity in patients with an acute attack of low back pain in a general practice setting. Spine 20(4): 478-484.

Kovacs F.M., Abraira V., Zamora J. and Fernandez C. 2005. The Spanish Back Pain Research Network. The transition from acute to subacute and chronic low back pain: a study based on determinants of quality of life and prediction of chronic disability. Spine 30: 1786-1792.

Lamers L.M., Meerding W.J., Severens J.L. and Brouwer W.B. 2005. The relationship between productivity and health related quality of life: An empirical exploration in persons with low back pain. Quality of Life Research 14: 805-813.

Majtas J. and Liscacova A. 2007. Quality of life assessment of pain treatment in diseases of back and the movement apparatus. Acta Facultis Pharmaceuticae Universiatis Comenianae 54: 124-135.

Maniadakis N. and Gray A. 2000. The economic burden of back pain in the UK. Pain 84: 95-103.

Moussouli M., Vlachopoulous S.P., Kofotolis N.D., Theodorakis Y., Malliou P. and Kellis E. 2014. Effect of stabilization exercise on health-related quality of life in women with chronic low back pain. Journal of Physical Activity and Health 11:1295-1303.

Ogunlana M.O., Odunaiya N.A., Dairo M.D. and Ihekuna O. 2012. Predictors of health-related quality of life in patients with non-specific low back pain. African Journal of Physiotherapy and Rehabilitation Sciences 2012; 4(1,2): 15-22.

Richardson C., Jull G., Hodges P. and Hides J. 1999. Therapeutic exercise for Spinal Segmental Stabilization in Low Back Pain: Scientific Basis and Clinical Approach. Edinburgh, NY: Churchill Livingstone.

Schiphorst Preuper H.R., Reneman M.F., Boonstra A.M., Dijkstra P.U., Versteegen G.J. and Geertzen J.H. 2008. Relationship between psychological factors and performance-based and selfreported disability in chronic low back pain. European Spine Journal 17: 1448-1456.

Waddell G., Feder G., Mclntosh A., Lewis M. and Hutchinson A.1996. Clinical Guidelines for Management of Acute Low Back Pain: Clinical guidelines and evidence review. London Royal College of General Practitioners.

Walker R.F., Muller R. and Grant W. 2004. Low back pain in Australian adults. Health provider utilization and care seeking. Journal of Manipulative Physiological Therapeutics 27: 327335.

Waxman R., Tennant A. and Helliwell P. 1998. Community survey of factors associated with consultation for low back pain. British Medical Journal 317: 1564-1567. 